\title{
Der digitale Fußabdruck, Schatten oder Zwilling von Maschinen und Menschen
}

\author{
Gregor Engels ${ }^{1}$ \\ Online publiziert: 12. August 2020 \\ (c) Der/die Autor(en) 2020
}

Digitale Technologien sind heutzutage in allen Bereichen unseres täglichen Lebens zu finden. Dies gilt für den privaten Bereich, in dem wir bevorzugt mit Smartphones und sozialen Medien kommunizieren, aber auch für den beruflichen Bereich, in dem digitale Maschinen wie Roboter unsere Produktionsprozesse oder Softwaresysteme unsere Arbeitsprozesse unterstützen. Hierbei ist ein wesentlicher Treiber einer stets fortschreitenden Digitalisierung, dass durch die immer höhere Verfügbarkeit des Internets in Echtzeit auch mit geographisch weit verteilten Beteiligten kommuniziert werden kann. Kommunikation und Austausch von Daten und damit auch Speicherung und Verarbeitung von Daten haben hierdurch einen enormen Stellenwert bekommen.

Eine große Menge von Daten ermöglicht es, mit maschinellen Lernverfahren der Künstlichen Intelligenz in diesen Daten enthaltenes Wissen zu extrahieren - etwa typische, immer wiederkehrende Verhaltensmuster von Maschinen oder auch von Menschen. Dieses abgeleitete Wissen kann bei verantwortungsvollem Einsatz etwa zur Verbesserung der medizinischen Versorgung oder zur Erhöhung der Effektivität von maschinellen Produktionsprozessen eingesetzt werden. Da es aber auch missbraucht werden kann, was insbesondere bei der Verarbeitung von personenbezogen Daten bedenkenswert ist, wurde in den letzten Jahren etwa durch die Einführung der Datenschutzgrundverordnung (DSGVO) viel Wert auf eine Verbesserung der gesetzlichen Regelungen im Umgang mit dem Datenschutz gelegt.

Ein wesentlicher Aspekt der fortschreitenden Digitalisierung ist also der Umgang mit Daten, die digital erfasst, gespeichert und verarbeitet werden können und in der Kommunikation mit anderen (digitalen) Partnern ausgetauscht

Prof. Dr. Gregor Engels

engels@upb.de

1 Institut für Informatik, Universität Paderborn, Warburger Str. 100, 33098 Paderborn, Deutschland werden können. Es ist eine der größten Herausforderungen unserer Zeit, mit diesen Daten verantwortungsbewusst umzugehen. Hierzu ist ein grundlegendes Verständnis von Aspekten der digitalen Verarbeitung von Daten notwendig. Denn jeder von uns produziert bei der Nutzung digitaler Technologien Daten (etwa beim Surfen, Chatten, Posten, Telefonieren im Internet), die dann gespeichert und weiterverarbeitet werden können. Diese digitalen Spuren, die man im Internet hinterlässt, können verfolgt und unterschiedlich intensiv genutzt werden. In diesem Beitrag sollen drei Formen näher erläutert werden: der digitale Fußabdruck, der digitale Schatten und schließlich der digitale Zwilling. Hierbei nehmen wir einen stark technisch geprägten Blickwinkel ein. Auf dieser Basis können dann Überlegungen angestellt werden, die auf der Basis einer werteorientierten Betrachtungsweise die Vorteile, aber auch Risiken dieser technologischen Machbarkeiten untersucht.

\section{Modell-getriebene Systementwicklung}

Unser Leben in der heutigen digitalisierten Welt ist geprägt durch soziotechnische Systeme (vgl. Maier et al. 2020). Derartige Systeme bestehen aus zwei wesentlichen Komponenten, einer technischen und einer sozialen Komponente. Zur technischen Komponente zählen materielle Dinge wie etwa Maschinen, aber auch immaterielle Dinge wie Softwaresysteme. Zur sozialen Komponente zählen Menschen, die die technischen Komponenten nutzen bzw. bedienen. In einem soziotechnischen System sind die beiden Teile nicht voneinander trennbar. Es bestehen enge Abhängigkeiten und beide Komponenten profitieren von einander durch verschiedene Formen der Kommunikation zwischen Menschen, zwischen Maschinen und vor allem zwischen Menschen und Maschinen, der sogenannten Mensch-MaschineInteraktion. Durch die Einbettung derartiger soziotechnischer Systeme in das Internet werden sie zu Cyber-Physical-Systems (CPS) bzw. bei Berücksichtigung der betei- 
ligten Menschen zu Socio-Cyber-Physical-Systems (SCPS) (Lee 2008).

Beispiele für derartige SCPS finden sich in allen Bereichen unseres Lebens. So etwa in der industriellen Produktion, wo Mitarbeiter*innen in kooperativer Weise mit Robotern arbeiten, in der Mobilität, wo Fahrer*innen mit durch Assistenzsysteme ausgerüstete Fahrzeuge nutzen oder in der Ausbildung, wo Internet-basierte Blended LearningTechnologien eingesetzt werden.

In der Softwaretechnik, einer Teildisziplin der Informatik, wurden in den letzten Jahren Konzepte und Methoden entwickelt, um derartige Systeme von hoher Qualität zu entwickeln und zu betreiben. Ein grundlegender Ansatz ist hierbei eine modell-basierte Vorgehensweise. Das bedeutet, dass vor Beginn der eigentlichen Realisierung auf Basis der ermittelten Anforderungen eines zukünftigen Nutzers des Systems ein Modell des Systems erstellt wird. Dieses Modell stellt eine Abstraktion eines Ausschnitts der realen Welt das und fokussiert auf die für das zu erstellende System relevanten Aspekte (Stachowiak 1973). Typische Aspekte sind hierbei die im System zu verwaltenden Objekt- und Datenstrukturen sowie Verarbeitungs- und Kommunikationsprozesse.

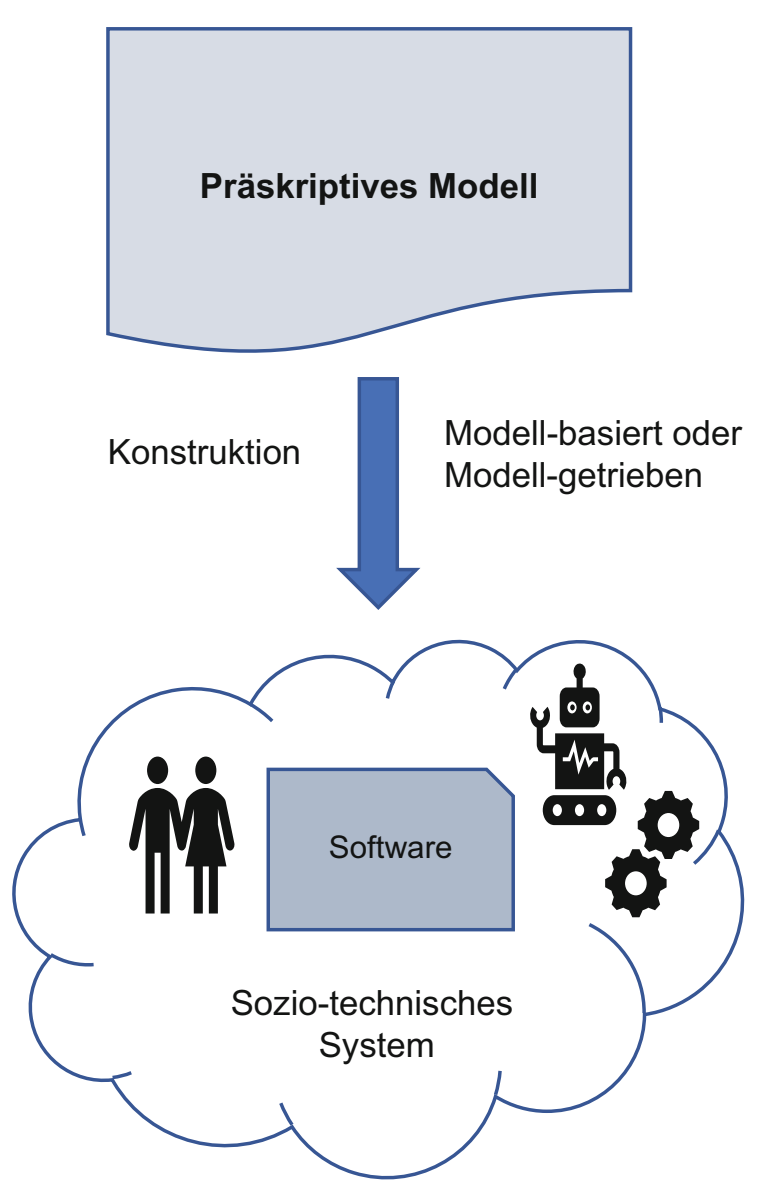

Abb. 1 Modell-basierte/Modell-getriebene Systementwicklung
Derartige Modelle werden mithilfe von standardisierten Modellierungssprachen wie etwa der UML (Unified Modeling Language) erstellt (vgl. UML 2005). Hier sind sowohl Syntax als auch Semantik eindeutig definiert, so dass bereits während der Erstellung des Modells syntaktische Korrektheitsanalysen und semantische Qualitätsanalysen durchgeführt werden können. Dies ist insbesondere bei großen Systemen von Vorteil, da bereits vor Beginn einer Programmierung Eigenschaften des Systems wie etwa eine sichere Datenkommunikation überprüft werden können.

In der Konstruktion des sozio-technischen Systems werden sowohl die materiellen Dinge als auch die immaterielle Software entwickelt (siehe Abb. 1). Aufgrund dieser über die Softwareentwicklung hinausgehenden Aufgaben spricht man hier mittlerweile in der Regel von einem Systems Engineering (vgl. Huth und Vietor 2020). Insbesondere im Bereich der Softwareentwicklung kann diese Konstruktion manuell (modell-basierte Entwicklung) oder automatisiert (modell-getriebene Entwicklung) erfolgen (Brambilla et al. 2019). Da das Modell in diesem Fall die Vorgabe für die Konstruktion ist, spricht man auch von einem präskriptiven Modell.

Aufgrund des Einsatzes von Software und der damit verbundenen „einfachen“ Änderbarkeit der Funktionalität der Software bieten Realisierungen von soziotechnischen Systemen die Möglichkeit, das System situativ an sich ändernde Nutzungs- und Umgebungsanforderungen anzupassen. Dies gilt etwa für die Mensch-Maschine-Interaktion, wo z.B. interaktive Assistenzsysteme an die unterschiedlichen Bedürfnisse und Fähigkeiten eines Nutzers angepasst werden können (Josifovska et al. 2019a). In der Informatik werden derartige Systeme adaptive Systeme genannt (Lemos et al. 2010). Sie heißen selbst-adaptiv, wenn eine derartige Anpassung vom System selbständig automatisiert durchgeführt wird.

Diese Fähigkeiten eines (selbst-)adaptiven Systeme spiegeln sich in der Verwendung einer Standard-Architektur wider, die unter der Bezeichnung MAPE-K bekannt ist (Kephart und Chess 2003) (siehe Abb. 2). MAPE-K ergänzt ein zu adaptierendes System um eine Adaptionskomponente. Mit einer Monitoring-Verbindung werden die für die Adaption relevanten Daten im zu adaptierenden System beobachtet und in einem zentralen Datenspeicher (Knowledge Base) abgelegt. Eine anschließende Analyse wertet die beobachteten Daten aus, indem sie etwa Vergleiche mit früher erhobenen Daten (auch anderer Systeme) ausführt, das Vorhandensein typischer Muster untersucht oder sie gemäß vorgegebener Parameter aggregiert. Die Ergebnisse werden wiederum im zentralen Datenspeicher abgelegt. Die anschließende Planung untersucht, ob und welche Veränderungen im System durchgeführt werden sollten. Sowohl in der Analyse als auch in der Planungsphase werden in der Regel Methoden der Künstlichen Intelligenz oder des Ope- 
Abb. 2 MAPE-K-Architektur für selbst-adaptive Systeme

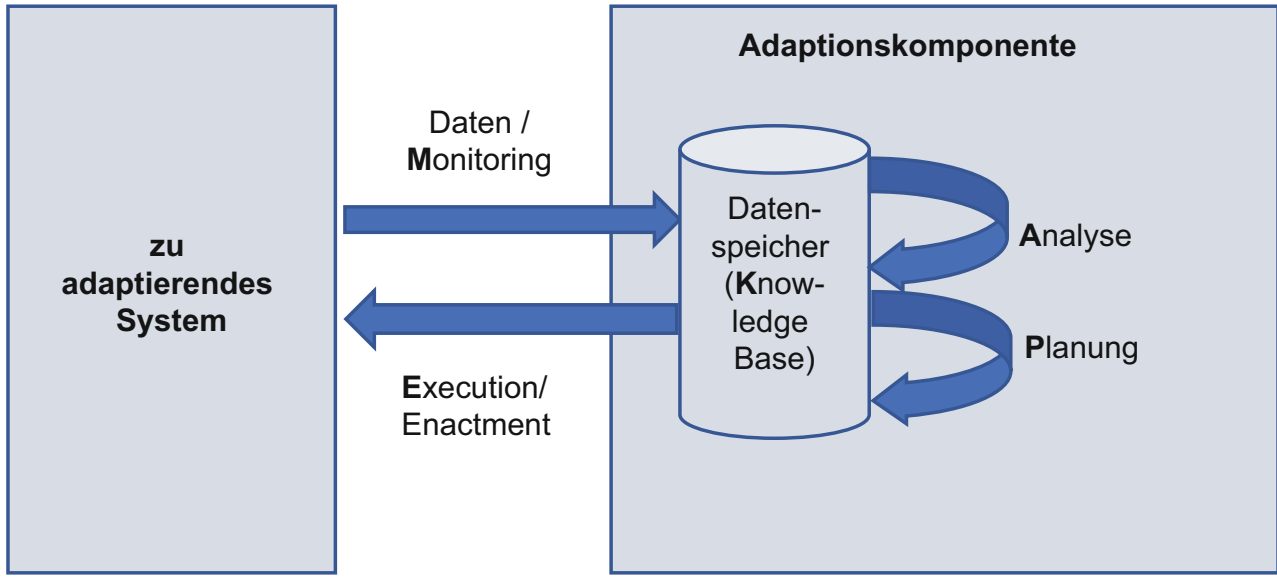

Ausfall eines Produktionssystems vorab zu erkennen und dementsprechend Gegenmaßnahmen zu ergreifen (,,predictive maintenance"),

- im Themenfeld Handel 4.0 erkannt, dass eine Analyse von Nutzerdaten helfen kann, Verkaufs- und Vermarktungsaktivitäten durch eine geeignete Individualisierung zu verbessern (,user profiling") (Eke et al. 2019),

- im Themenfeld Unternehmen 4.0 erkannt, dass ein besseres Verständnis der Kompetenzen und auch der gesundheitlichen Verfassung von Mitarbeiter*innen Effizienz und Effektivität beim Einsatz einzelner Mitarbeiter in einem Team erhöhen kann (,employee monitoring“).

Konkrete Beispiele sind etwa im Themenfeld Industrie 4.0 die Ankündigung der Volkswagen AG, dass eine von Siemens entwickelte Software die mehr als 120 Volkswagen-Werke weltweit miteinander vernetzen und damit die technische Grundlage für geplante Produktivitätsfortschritte schaffen soll (vgl. Süddeutsche Zeitung 2019). Hierbei sollen etwa Verbrauchs- und Produktionsdaten von mehreren hunderttausend Maschinen erfasst, gespeichert und anschließend analysiert werden. Hierdurch können Ausfall- und Wartezeiten im Produktionsprozess reduziert werden (,predictive maintenance“) und Produktionsprozesse optimiert werden.

In ähnlicher Form ist es im Bereich Handel 4.0, in dem Firmen wie Amazon, Google oder Facebook Daten über einzelne Nutzer speichern und analysieren (Gläß und Leukert 2016). Hierbei werden z.B. beim Einsatz von Smartphones nicht nur Daten über besuchte Webseiten auf der Basis der Markierung durch Cookies gesammelt, sondern auch Angaben über genutzte Browserversionen, installierte Apps oder auch Orts- und Bewegungsdaten (,,bowser fingerprint") gesammelt. Durch Analyse dieser Daten werden von Werbefirmen Profile einzelner Nutzer ermittelt und entsprechend personalisierte Produktwerbungen etwa auf einem Smartphone angezeigt (vgl. TheConversation 2019).

- im Themenfeld Industrie 4.0 erkannt, dass eine Analyse von Produktionsdaten helfen kann, einen möglichen 
Schließlich können Unternehmen heutzutage die Daten nutzen, die in sozialen Unternehmensnetzwerken (,Enterprise Social Networks“) wie z.B. MyAnalytics von Microsoft (MyAnalytics 2020) oder Chatter von Salesforce (Chatter 2020) erhoben werden, um Informationen über die Aktivitäten von Mitarbeiter*innen am Arbeitsplatz wie z. B. der prozentuale Arbeitszeitanteil im E-Mail-Bearbeiten, im Telefonieren, in Videokonferenzen oder Chats oder auch die gepflegten Kontaktbeziehungen zu erhalten.

Diese unterschiedlichen Szenarien haben alle das Ziel, Daten von Menschen, Maschinen und eingesetzter Software während der Nutzung eines soziotechnischen Systems aufzunehmen, zu speichern, zu analysieren und als Grundlage einer weiteren Verarbeitung zu nutzen. In all diesen Fällen stellt sich die Frage, wem die Daten gehören und wer sie nutzen, auswerten und weitergeben darf, so dass aufgrund dieser hohen Verfügbarkeit von Daten Fragen des Datenschutzes, der Datenautonomie, aber auch etwa des Arbeitsrechts einen immer höheren Stellenwert bekommen.

Zielsetzung dieses Beitrags ist nun im Folgenden die Darstellung eines konzeptionellen Rahmenwerks für unterschiedliche Formen der Aufnahme und Verarbeitung von maschinen- bzw. personenbezogenen Daten während der Ausführung eines soziotechnischen Systems. Auf dieser Basis können dann weiterführende Diskussionen geführt werden. Hierzu gehören dann auch Fragen des Schutzes von personenbezogenen Daten, aber auch weitergehende Fragen der Auswirkungen des Einsatzes von datenbasierten Verfahren zur Beeinflussung und Steuerung von soziotechnischen Systemen.

\section{Digitale Lösungen: Fußabdruck, Schatten, Zwilling}

Jede Aktivität in einem Internet-basierten soziotechnischen System führt zu Bewegungsdaten, die beobachtet und gespeichert werden können und auf dieser Basis zu einer Reaktion des Systems führen können. Hierbei kann unterschieden werden

1. ob bei identitätsbezogenen Daten im Nachhinein die einzelne Person oder Maschine identifiziert werden kann,

2. ob die Daten passiv (ohne Wissen der beobachteten Person bzw. Maschine) erhoben wurden oder die Verbreitung und damit ermöglichte Erhebung aktiv von den Beteiligten durchgeführt wurde,

3. ob die Daten individualisiert oder aggregiert ausgewertet werden,

4. ob die Daten in Echtzeit oder zu einem späteren Zeitpunkt ausgewertet werden,

5. ob bei individualiserten, insbesondere personenbezogenen Daten die betroffene Person die Entscheidungshoheit über den Zugriff behält,

6. ob die beobachteten und gespeicherten Daten einen oder mehrere Aspekte eines realen soziotechnischen Systems umfänglich abbilden.

Um diese Unterschiede einordnen zu können, wird das in Abb. 3 dargestellte Rahmenwerk vorgestellt. Es beruht auf dem oben eingeführten Architekturmuster MAPE-K für selbst-adaptive Systeme sowie auf dem MODA-Framework, einem Framework, welches das Zusammenspiel von Modellen, Systemen und Daten in einen Zusammenhang stellt (Combemale et al. 2020).
Abb. 3 Konzeptionelles Rahmenwerk zur Einordnung der Begriffe Digitaler Fußabdruck, Digitaler Schatten und Digitaler Zwilling
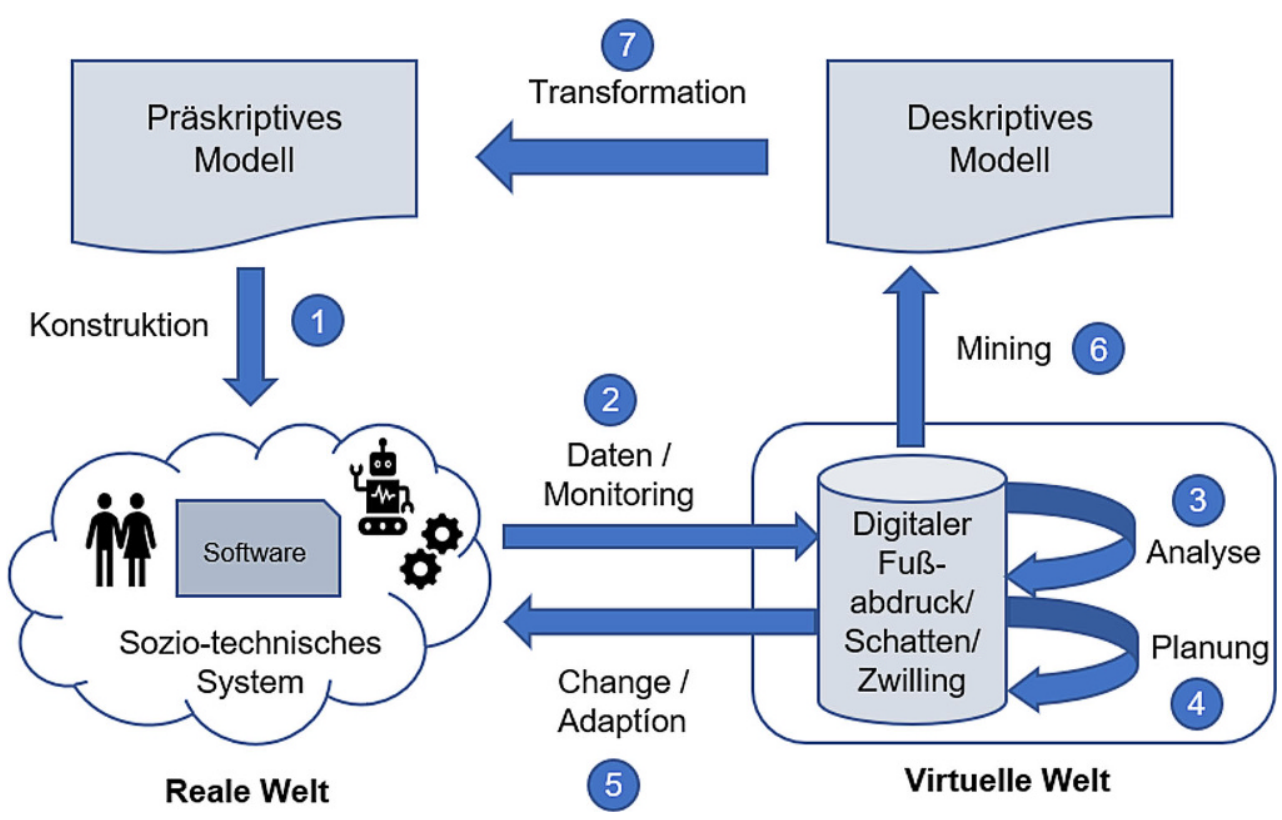

Virtuelle Welt 
Ausgehend von einer Konstruktion eines soziotechnischen Systems (Schritt 1) können bei einer Ausführung des Systems Daten aktiv in der virtuellen Welt (etwa durch Veröffentlichen in sozialen Medien) oder passiv (etwa durch Beobachten (Monitoring) des Kommunikationsverkehrs (Schritt 2)) in einem zentralen Datenspeicher abgelegt werden. Hierbei ist zu beachten, dass der Datenspeicher sehr umfangreich sein kann und eine stark vernetzte Struktur haben kann, da hier sowohl Daten einzelner Elemente eines soziotechnischen Systems abgelegt sind als auch die Daten mehrerer soziotechnischer Systeme abgelegt sein können. Der Datenspeicher ist somit eine zentrale Instanz, die in den Schritten 3 und 4 die Grundlage für eine Analyse und eine weitere Planung bildet. Aufgrund des großen Umfangs von Daten können hier erfolgreich maschinelle Lernverfahren der Künstlichen Intelligenz eingesetzt werden, um etwa typische Muster und Abhängigkeiten in den Daten zu erkennen und auf dieser Basis im Planungsschritt Veränderungen am soziotechnischen System vorzubereiten.

Ein konkretes Beispiel sind etwa selbst-adaptive Assistenzsysteme für Mitarbeiter*innen in der industriellen Produktion, bei denen das Verhalten eines/r Mitarbeiter*in analysiert und mit ermittelten Verhaltensmustern verglichen wird und daraufhin eine veränderte Funktionalität etwa in Form einer stärkeren Nutzerführung im Assistenzsystem umgesetzt wird (Josifovska et al. 2019a).

Diese Umsetzung im realen soziotechnischen System geschieht dann im Schritt 5. Die Schritte 2-5 beschreiben somit die Feedbackschleife eines adaptiven soziotechnischen Systems.

Auf diese Art und Weise können vorab geplante Änderungen am realen soziotechnischen System vorgenommen werden. Eine tiefergehendere Änderung und damit Weiterentwicklung des soziotechnischen Systems kann erfolgen, wenn die im Datenspeicher abgelegten Daten einen bestimmten Aspekt des realen Systems (etwa Betriebseigenschaften einer Maschine oder Profildaten eines Mitarbeiters) umfänglich abbilden. In diesem Fall kann mit Hilfe von Data-Mining-Techniken (Schritt 6) von den vorliegenden Daten ein deskriptives Modell dieses Wissens abgeleitet werden. Ein typisches Beispiel sind etwa Process Mining Techniken, die erfasste Aktivitätsprozesse von Menschen oder Maschinen analyiseren und in einem abstrakten
Prozessmodell ablegen (Van der Aalst 2012). Im nächsten Schritt 7 kann auf dieser Basis das präskriptive Modell des Systems aktualisiert werden, in dem dann etwa die Erkenntnisse über veränderte Produktions- oder Arbeitsprozesse unterstützt werden. So entsteht dann daraus eine neue Version eines adaptiven soziotechnischen Systems (Schritt 1). Hierdurch schließt sich eine zweite Feedback-Schleife, mit der schrittweise neue Systemversionen realisiert werden.

Es gibt in der Literatur eine umfangreiche Liste von Referenzen zur Definition und zum Einsatz des digitalen Zwillings in der industriellen Produktion (Grieves und Vickers 2017; Tao und Zhang 2017; Uhlemann et al. 2017; Vachálek et al. 2017), die auf der ursprünglichen Definition von Grieves (2004) aufbauen. Hier wird der digitale Zwilling als ein digitales Abbild einzelner Maschinen definiert, wobei hierzu nicht nur charakterisierende Attribute gehören, sondern auch typische Funktionalitäten dargestellt durch Algorithmen. Mit ihnen kann man dann das Verhalten des realen Objeks simulieren und hierdurch Eigenschaften erproben oder vorausplanen.

Da zu einem soziotechnischen System neben den Maschinenobjekten auch Menschen gehören, ist es naheliegend auch über den digitalen Zwilling eines Menschen nachzudenken. Hierzu gibt es in der Literatur noch kein einheitliches Verständnis und dementsprechend auch keine einheitliche Definition (Feher 2019). Wie bereits oben erläutert, werden auch über Menschen umfangreiche Daten digital gesammelt, die dann unter den Begriffen Digitaler Fußabdruck (,digital footprint“) oder Digitaler Schatten (,digital shadow") diskutiert werden. Hierbei ist der Übergang zwischen diesen beiden Begriffen fließend. Häufig werden sie auch synonym genutzt.

Wichtig ist hier zu verstehen, dass es bei dem digitalen Zwilling des Menschen nicht darum geht, Menschen zu klonen oder wie etwa im europäischen Human Brain Project (HBP) das menschliche Gehirn mittels computerbasierter Modelle und Simulationen nachzubilden (HBP 2020). Es geht hier darum, für eine bestimmte Situation relevante charakterisierende Eigenschaften eines Menschen zu speichern und zu analysieren. Hierzu gehören dann z.B. demographische oder physiologische Daten oder Daten zum Kompetenz- oder Tätigkeitsprofil bzw. zum Gesundheitszustand eines Menschen.

Tab. 1 Merkmale zur Charakterisierung der drei Begriffe Digitaler Fußabdruck, Digitaler Schatten, Digitaler Zwilling

\begin{tabular}{llll}
\hline Merkmale & Digitaler Fußabdruck & Digitaler Schatten & Digitaler Zwilling \\
\hline 1. Identifzierbarkeit & (Ja)/Nein & Ja & Ja \\
2. Passiv/Aktiv & Passiv/Aktiv & Passiv/Aktiv & Passiv/Aktiv \\
3. Individualisierte Auswertung & Nein & $(\mathrm{Ja}) /$ Nein & Ja \\
4. Echzeitauswertung & Nein & Nein & Ja \\
5. Entscheidungshoheit & Nein & Nein & (Nein)/Ja \\
6. Umfängliche Abbildung & Nein & Nein & Ja \\
\hline
\end{tabular}


Tab. 2 Charakterisierung anhand vorgegebener Merkmale (siehe Tab. 1)

\begin{tabular}{lllll}
\hline & Internetrecherche & Gebäude 4.0 & Industrie 4.0 & Arbeit 4.0 \\
Merkmale & Digitaler Fußabdruck & Digitaler Schatten & Digitaler Zwilling & Digitaler Zwilling \\
\hline 1. Identifzierbarkeit & Nein & Ja & Ja & Ja \\
2. Passiv/Aktiv & Passiv & Aktiv & Aktiv & Passiv/Aktiv \\
3. Individualisierte Auswertung & Nein & Ja & Ja & Ja \\
4. Echzeitauswertung & Nein & Nein & Ja & Ja \\
5. Entscheidungshoheit & Nein & Nein & n.z. & (Nein)/Ja \\
6. Umfängliche Abbildung & Nein & Nein & Ja & (Ja) \\
\hline
\end{tabular}

Da es in der Literatur keine einheitliche Definition der drei Begriffe digitaler Fußabdruck, digitaler Schatten bzw. digitaler Zwilling insbesondere zum Menschen gibt, werden diese drei Begriffe im Folgenden (siehe Tab. 1) anhand der oben erläuterten Merkmale charakterisiert. Die verwendeten Merkmale wurden u.a. aus ersten existierenden systematischen Literaturstudien abgeleitet (Josifovska et al. 2019b; Van der Valk et al. 2020):

Diese Charakterisierung besagt, dass bei einem digitalen Fußabdruck eine Anzahl von Daten gespeichert werden, die aber in der Regel keine Identifizierbarkeit der einzelnen Maschine oder der einzelnen Person zulassen. Eine häufig genutzte Technik ist das Platzieren von Cookies in der verwendeten Internettechnologie (etwa Webbrowser), die dann bei einem erneuten Besuch einer Webseite Informationen über vergangene Zugriffe enthalten. In diesem Sinne wird in diesem Fall auch nur Schritt 2 des Rahmenwerks (s. Abb. 3) genutzt.

Dies unterscheidet sich grundsätzlich von einem digitalen Schatten oder sogar einem digitalen Zwilling, wo es darum geht, beobachtete Daten einer konkreten Maschine oder Person zuordnen zu können. Hier werden die Daten in der Regel auch analysiert (Schritt 3). Allerdings wird erst bei einem digitalen Schatten in Echtzeit eine unmittelbare Verbindung zum System hergestellt (Schritt 5), nachdem vorher geplant wurde (Schritt 4), wie auf eine Analyse der Daten zu reagieren ist. Ein Beispiel sind hier etwa adaptive Assistenzsysteme für Mitarbeiter*innen, bei denen das Verhalten einer Mitarbeiter*in beobachtet, analysiert und entsprechend etwa durch Anpassung eines Betriebsablaufs reagiert wird.

Weiterhin grenzt sich ein digitaler Zwilling dadurch ab, dass für die aktuelle Situation eine umfängliche Sicht auf einen Menschen gespeichert und verarbeitet wird. Hierbei geschieht die Aktualisierung dieser Datenspeicherung in Echtzeit, so dass man erst hier von einem Zwilling reden kann. Dies erlaubt dann auch eine individualisierte, auf den einzelnen Menschen bezogene Auswertung. Inwieweit der Mensch die Entscheidungshoheit behält, wer einen Zugriff auf die digitalen Daten hat, ist im Einzelfall zu entscheiden. Ein aktuelles Beispiel ist hier die Einführung der elektronischen Gesundheitskarte, bei der zu entscheiden ist, ob Da- ten allen behandelnden Ärzten zur Verfügung stehen oder ob man hier Leserechte einschränken kann. Ein digitaler Zwilling liegt allerdings hier nicht vor, da die Daten auf der Gesundheitskarte nicht in Echtzeit aktualisiert werden.

Durch eine Vernetzung der Daten von vielen digitalen Zwillingen können übergreifende Muster und Eigenschaften in diesen Daten sowohl zu einem aktuellen Zeitpunkt als auch im Verlauf der Zeit analysiert werden.

\section{Anwendungsszenarien: Fußabdruck, Schatten, Zwilling}

In diesem Abschnitt erläutern wir einige typische Anwendungen und charakterisieren sie anhand der oben genannten Merkmale als digitalen Fußabdruck, digitalen Schatten oder digitalen Zwilling (siehe Tab. 2).

Internetrecherche Einen digitalen Fußabdruck findet man vor allem bei jeder Form von Internetrecherche. Hier werden in der Regel keine personenbezogenen Identitätsdaten gespeichert und auch kein umfassendes digitales Abbild einer einzelnen Person abgelegt. Mit Unterstützung technischer Hilfsmittel wie Cookies werden dennoch Verläufe und Häufigkeit etwa von Webseitenzugriffen ermittelt und ausgewertet.

Gebäude 4.0 Einen Bereich mit wachsender Bedeutung für den Einsatz eines digitalen Schattens findet man etwa im Baugewerbe. Durch Nutzung des Building-Information-Model-(BIM-)Ansatzes können Gebäude in digitaler Form modelliert und abgelegt werden (ISO 19650-1 2018). Dieses Vorgehen erleichtert die Integration der Gewerke in der Gebäudebranche insbesondere bei der Wartung und Veränderung von Gebäuden, da in dem BIM-Modell alle gebäuderelevanten Daten abgelegt sind. Zurzeit wird an der Realisierung eines solchen Systems für die Gebäudeerstellung gearbeitet (Rothenbusch und Kauffeld 2020). Auch durch die Verwendung digitaler Strom- und Wasserzähler entsteht im Laufe der Zeit ein digitaler Schatten einzelner Häuser, der von Versorgungs- und Stadtplanungsunternehmen genutzt werden kann. 
Industrie 4.0 In diesem Anwendungsgebiet wurde sehr früh der Begriff des digitalen Zwillings für Maschinen geprägt. Hierbei war die Intention, ein möglichst vollständiges Abbild einer konkreten Maschine zu erhalten, um Betriebsdaten zu speichern, mögliche Abläufe vorab zu simulieren und in Echtzeit reagieren zu können. Neben einer individualisierten Auswertung werden auch aggregierte Auswertungen durchgeführt, um etwa Informationen über den Betrieb eines bestimmten Maschinentyps zu erhalten.

Arbeit 4.0 In Erweiterung der Diskussion im Gebiet Industrie 4.0 wurde in den letzten Jahren auch intensiv die Rolle von Arbeitnehmer*innen unter dem Thema Arbeit 4.0 untersucht (Maier et al. 2020). Auch hier wurde vorgeschlagen, einen digitalen Zwilling von Arbeitnehmer*innen zu erstellen, um ihre Situation am Arbeitsplatz zu beobachten und durch Anpassung der Arbeitsmittel zu verbessern. Es ist hierbei erstrebenswert, dass die Entscheidungshoheit bei dem/r Mitarbeiter*in liegt, von dem/r die Daten erhoben wurden. Im Sinne einer umfänglichen Abbildung ist bei einem digitalen Zwilling eines/r Arbeitnehmer*in nicht beabsichtigt, einen Menschen vollständig abzubilden. Es geht hier um die relevanten, arbeitsplatzbezogenen Attribute eines/r Mitarbeiter*in wie etwa Ausbildungsstand, aber durchaus auch Zufriedenheits- und Gesundheitsattribute.

Auch in anderen Bereichen wird das Konzept eines digitalen Schattens bzw. eines digitalen Zwillings schrittweise eingeführt. Dies gilt etwa für Diskussionen im Bereich der öffentlichen Verwaltung (eGovernment) oder im Bereich der Gesundheitsversorgung (elektronische Gesundheitskarte).

\section{Zusammenfassung und Ausblick}

Die Sammlung, Speicherung und Verarbeitung von Daten ist aufgrund der immer umfangreicheren technischen Möglichkeiten ein wichtiges Thema. In diesem Beitrag wurden einige grundlegende konzeptionelle Überlegungen dargestellt und ein charakterisierendes Rahmenwerk eingeführt. Dies soll helfen, die Diskussionen und die weitere Entwicklung rund um den digitalen Zwilling einordnen zu können.

Hierzu gehört etwa die Diskussion, wie in Zukunft Daten erhoben werden. Videoüberwachungen, automatisierte Gesichtserkennungen, Mikrochips unter der Haut sind neue Formen der Datenerfassung, die technisch möglich sind, aber auch viele Risiken (z. B. Fehler in der Erkennungssoftware, unkontrollierter Zugriff auf personenbezogene Daten) mit sich bringen.

Bei der Speicherung der Daten stellt sich die Frage, inwieweit hier verschiedene Datenbestände integriert werden dürfen. Hierdurch würde eine viel größere Wissensbasis für Analysealgorithmen entstehen, die die Erkennung bisher nicht identifizierter Zusammenhänge und Abhängigkeiten ermöglicht. Dies ist ein wichtiges Thema etwa in der Gesundheitsforschung (z. B. Einfluss von Umweltbedingungen auf die Häufigkeit von bestimmten Krankheitsfällen) oder in der Kriminalitätsverfolgung (grenzüberschreitender Abgleich von Kriminalitätsdaten).

Wo sind die rechtlichen, aber auch ethischen Grenzen einer derartigen datenbasierten Gesellschaft? Sollen die Daten genutzt werden, um Unternehmens- und Gesellschaftsstrukturen zu unterstützen oder zu kontrollieren. Hierzu gehört etwa die Diskussion rund um das Chinese Social Credit System, in dem zu jedem chinesischen Bürger eine elektronische Akte in Form eines digitalen Zwillings geführt wird, in der positive, aber auch im Sinne der Regierung negative Aspekte eines Einzelnen gespeichert und für Entscheidungen im privaten und beruflichen Umfeld genutzt werden (New Horizons 2020).

In diesem Sinne muss eine gesamtgesellschaftliche, Wissenschaftsdisziplinen-übergreifende Diskussion geführt werden, damit die Chancen einer datenbasierten Gesellschaft genutzt werden und die Risiken und Gefahren minimiert werden.

Funding Open Access funding provided by Projekt DEAL.

Open Access Dieser Artikel wird unter der Creative Commons Namensnennung 4.0 International Lizenz veröffentlicht, welche die Nutzung, Vervielfältigung, Bearbeitung, Verbreitung und Wiedergabe in jeglichem Medium und Format erlaubt, sofern Sie den/die ursprünglichen Autor(en) und die Quelle ordnungsgemäß nennen, einen Link zur Creative Commons Lizenz beifügen und angeben, ob Änderungen vorgenommen wurden.

Die in diesem Artikel enthaltenen Bilder und sonstiges Drittmaterial unterliegen ebenfalls der genannten Creative Commons Lizenz, sofern sich aus der Abbildungslegende nichts anderes ergibt. Sofern das betreffende Material nicht unter der genannten Creative Commons Lizenz steht und die betreffende Handlung nicht nach gesetzlichen Vorschriften erlaubt ist, ist für die oben aufgeführten Weiterverwendungen des Materials die Einwilligung des jeweiligen Rechteinhabers einzuholen.

Weitere Details zur Lizenz entnehmen Sie bitte der Lizenzinformation auf http://creativecommons.org/licenses/by/4.0/deed.de.

\section{Literatur}

Brambilla, M., Cabot, J., \& Wimmer, M. (2019). Model-driven software engineering in practice (2. Aufl.). San Rafael: Morgan \& Claypool.

Combemale, C., Kienzle, J., Mussbacher, G., Ali, H., Amyot, D., Bagherzadeh, M., Batot, E., Bencomo, N., Benni, B., Bruel, J.M., Cabot, J., Cheng, B., Collet, P., Engels, G., Heinrich, R., Jézéquel, J.-M., Koziolek, A., Mosser, S., Reussner, R., Sahraoui, H., Rijul Sainiy, R., Sallou, J., Stinckwich, S., Syriani, E., \& Wimmer, M. (2020). A hitchhiker's guide to model-driven engineering for data-centric systems. IEEE Software. Im Druck.

Eke, C. I., Norman, A. A., Shuib, L., \& Nweke, H. F. (2019). A survey of user profiling: state-of-the-art, challenges, and solutions. IEEE Access, 7, 144907-144924. 
Feher, K. (2019). Digital identity and the online self: footprint strategies - an exploratory and comparative research study. Journal of Information Science. https://doi.org/10.1177/0165551519879702.

Gläß, R., \& Leukert, B. (Hrsg.). (2016). Handel 4.0: Die Digitalisierung des Handels - Strategien, Technologien, Transformation. Berlin, Heidelberg: Springer Gabler.

Grieves, M. (2004). Digital twin-manufacturing excellence through virtual factory replication. Whitepaper. Florida Institute of Technology: LLC.

Grieves, M., \& Vickers, J. (2017). Digital twin: mitigating unpredictable, undesirable emergent behavior in complex systems. In F.J. Kahlen, S. Flumerfelt \& A. Alves (Hrsg.), Transdisciplinary perspectives on complex systems (S. 85-113). Cham: Springer.

HBP (2020). Human brain project. https://www.humanbrainproject.eu/ en/. Zugegriffen: 13. Juli 2020.

Huth, T., \& Vietor, T. (2020). Systems Engineering in der Produktentwicklung: Verständnis, Theorie und Praxis aus ingenieurswissenschaftlicher Sicht. Gruppe.Interaktion.Organisation, Zeitschrift für Angewandte Organisationspsychologie (GIO), 51, 125-130.

ISO 19650-1:2018 (2018). Organization and digitization of information about buildings and civil engineering works, including building information modelling (BIM)_Information management using building information modelling-Part 1: Concepts and principles

Josifovska, K., Yigitbas, E., \& Engels, G. (2019a). A digital twin-based multi-modal UI adaptation framework for assistance systems in industry 4.0. In: Proceedings of the 21st International Conference on Human-Computer Interaction (HCII 2019). (S. 398-409). Cham: Springer.

Josifovska, K., Yigitbas, E., \& Engels, G. (2019b). Reference framework for digital twins within cyber-physical systems. IEEE/ACM 2019 (S. 25-31). https://ieeexplore.iee.org/xpl/conhome/ 8812309/proceeding.

Kephart, J. O., \& Chess, D. M. (2003). The vision of autonomic computing. Computer, 36(1), 41-50.

Lee, E. A. (2008). Cyber physical systems: design challenges. In 11th IEEE International Symposium on Object and Component-Oriented Real-Time Distributed Computing (ISORC) (S. 363-369). Piscataway: IEEE.

Lemos, R., Giese, H., Müller, H. A., Shaw, M., Andersson, J., Litoiu, M., Schmerl, B. R., Tamura, G., Villegas, N. M., Vogel, T., Weyns, D., Luciano Baresi, L., Becker, B., Bencomo, N., Brun, Y., Cukic, B., Desmarais, R. J., Dustdar, S., Engels, G., Geihs, K., Göschka, K. M., Gorla, A., Grassi, V., Inverardi, P., Karsai, G., Kramer, J., Lopes, A., Magee, J., Malek, S., Mankovski, S., Mirandola, R., Mylopoulos, J., Nierstrasz, O., Pezzè, M., Prehofer, C., Schäfer, W., Schlichting, R.D., Smith, D.B., Sousa, J.P., Tahvildari, L., Wong, K., \& Wuttke, J. (2010). Software engineering for selfadaptive systems: a second research roadmap. In R. de Lemos, et al. (Hrsg.), Software engineering for self-adaptive systems II (S. 1-32). Berlin, Heidelberg: Springer.

Maier, G. W., Engels, G., \& Steffen, E. (2020). Einleitung. In G. W. Maier, G. Engels \& S. Steffen (Hrsg.), Handbuch Gestaltung digitaler und vernetzter Arbeitswelten. Berlin: Springer.

Microsoft (2020). MyAnalytics. https://www.microsoft.com/en/micro soft-365/business/myanalytics-personal-analytics. Zugegriffen: 13. Juli 2020.
New Horizons (2020). An introduction to China's social corporate credit system. https://nhglobalpartners.com/chinas-social-creditsystem-explained/. Zugegriffen: 13. Juli 2020.

Rothenbusch, S., \& Kauffeld, S. (2020). Auswirkungen der Digitalisierung des Planungs- und Ausführungsmanagement auf kooperative Arbeitsprozesse im Bauhandwerk. Gruppe.interaktion.organisation. Zeitschrift Für Angewandte Organisationspsychologie.

Salesforce (2020). Chatter. https://www.salesforce.com/eu/products/ chatter/overview/. Zugegriffen: 13. Juli 2020.

Stachowiak, H. (1973). Allgemeine Modelltheorie. Wien, New York: Springer.

Süddeutsche Zeitung (2019). Digitale Fabrik. https://www.sued deutsche.de/wirtschaft/digitale-fabrik-siemens-soll-vw-werke-ver netzen-1.4389311. Zugegriffen: 13. Juli 2020.

Tao, F., \& Zhang, M. (2017). Digital twin shop-floor: a new shopfloor paradigm towards smart manufacturing. IEEE Access, 5, 20418-20427.

TheConversation (2019). Amazon, Facebook and Google don't need to spy on your conversations to know what you're talking about. https://theconversation.com/amazon-facebook-and-google-dontneed-to-spy-on-your-conversations-to-know-what-youre-talkingabout-108792. Zugegriffen: 13. Juli 2020.

Uhlemann, T. H. J., Lehmann, C., \& Steinhilper, R. (2017). The digital twin: realizing the cyber-physical production system for industry 4.0. Procedia Cirp, 61, 335-340.

UML (2005). Unified Modeling Language, Object Management Group. https://www.omg.org/spec/UML/. Zugegriffen: 13. Juli 2020.

Vachálek, J., Bartalsky, L., Rovny, O., Sismisova, D., Morhác, M., \& Loksík, M. (2017). The digital twin of an industrial production line within the industry 4.0 concept. In Process Control (PC), 21 st International Conference (S. 258-262). Piscataway: IEEE.

Van der Aalst, W. (2012). Process mining. Communications of the ACM, 55(8), 76-83.

Van der Valk, H., Haße, H., Möller, F., Arbter, M., Henning, J.-L., \& Otto, B. (2020). A Taxonomy of Digital Twins. In 26th Americas Conference on Information Systems (AMCIS). Salt Lake City, USA.

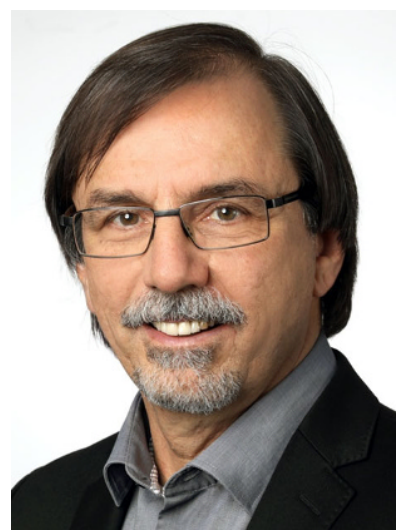

Prof. Dr. Gregor Engels ist Professor für Informatik an der Universität Paderborn. Er ist Sprecher des NRW Forschungskollegs „Gestaltung von flexiblen Arbeitswelten“" sowie des Forschungsschwerpunkts „Digitale Zukunft“ (gemeinsam mit der Universität Bielefeld). Er leitet den Software Innovation Campus Paderborn (SICP) und forscht im Bereich der modellbasierten Softwareentwicklung. 